\section{Filling in} space

\section{C.W. Kilmister}

Relativity and Geometry.

By Roberto Torretti.

Pergamon: 1983. Pp.395. £22.50, \$45.

A PERENNIAL trouble with the philosophy of science, and, to slightly lesser extent, its history, is that of finding an adequate level of philosophical and historical scholarship in those trained fully to understand the science and vice versa. It is a joy to record how, in this "historico-critical exposition" of special and general relativity, Roberto Torretti triumphs over this problem.

The story begins with Newton, who is seen as the author of the revolution against Aristotle, but who also went wrong by assuming, in a natural seventeenth-century way, that featureless Euclidean space must be endowed with mechanical existence. This extra assumption then involved him in the need for an absolute space, just as he had already followed Aristotle in requiring an absolute time. Not that Newton had no choice for, since Elie Cartan in 1923, we can formulate mechanics against an appropriate Newtonian space-time. But the mathematical description of this involves such highly twentieth-century concepts that it is difficult to believe that even Newton could have done it had he wanted. (Of course, he did not want to, for he had sound theological reasons for preferring an absolute space.)

We move on to the familiar tale of electromagnetism and the aether, through Maxwell, to Einstein's 1905 paper which is dealt with at length. A thorough discussion is given, too, of Whittaker's mythical "Relativity Theory of Poincare and Lorentz". The following chapter, just as long, on Minkowski space-time includes, as well as more familiar material, consideration of Robb's theory of a causal structure for space-time and also Zeeman's striking connection between the causal and the affine metric structures (that the group of causal automorphisms is the group of orthochronous similarities). An important role here is played by the concept of a causal space in the sense of Kronheimer and Penrose. Minkowski space-time is such a space, but Newtonian space-time fails to satisfy the requirement that any neighbourhood of a point contains a causal curve through the point. Robb's achievement was then to show that the same axioms that defined the causal structure of Minkowski space-time were sufficient to specify its affine and metric structures (up to a dilation).

Next there is a description of Einstein's search for a theory of gravitation, due attention being given to the curious papers of 1907 and 1911 (where a variable velocity of light serves as the gravitational poten- tial). Here there is also a notably sympathetic treatment of Nordstrøm's 1912 theory, which inspired Einstein and Fokker to reformulate it in 1914, and may perhaps have stimulated Einstein's physical thinking in just the way that was needed to allow him to appreciate the ideas of Marcel Grossmann. The description of general relativity goes beyond 1915 to discuss the problem of the equations of motion and the related one of gravitational radiation.

Torretti then proceeds to gravitational geometry, seeking to "elucidate a few matters that I consider especially significant from a philosophical point of view" - the role of the conformal structure of space-time, Mach's principle and cosmology, singularities and the main singularity theorems - before tackling what he calls "disputed questions". One of these is the definition of simultaneity (in special relativity), and, from that, the discussion of geometric conventionalism. Here Poincaré is not mentioned but Reichenbach is, getting short shrift for his misconstrual of gravity. Grünbaum, on the other hand, is castigated for obscurity. Finally questions of time and causality are discussed.

\section{Upper crusts}

\section{Janet V. Watson}

\section{Mountain Building Processes.}

Edited by Kenneth J. Hsü.

Academic: 1983. Pp.263. £39.40, \$72.50.

JUST another symposium volume on a plate tectonic theme? No, this book is different. The background seems quite familiar: a meeting in Zürich in 1981; a theme complex enough to arouse interest but not so vast as to seem formless; a couple of dozen contributors, neatly mixing the acknowledged masters with the young turks; and a dedicated editor who knows how to wheedle acceptable manuscripts out of his participants. Many useful but dull volumes have been built on just such a formula. What makes this one so enjoyable?

A clue is to be found in Professor Hsü's introduction, in which he uses the image of the anatomist reconstructing a living organism from a skeleton to illustrate the geologist's approach to mountain belts. The exhilaration which the authors have felt in breathing life into their subject comes through in article after article. A glance at the list of contributors - and at the photographs of Augusto Gansser and Rudolph Trümpy to whom the book is dedicated - shows that many of them are people with a lifetime's experience of mountains, people who have talked mountain-building in tents and cafés and landrovers all over the world. Moreover, they share a common interest in the interpretation of three-dimensional structures, an art fundamental to tectonic studies.

Another clue comes from the plan of the
An appendix gives a modern, largely coordinate-free, description of manifolds, fibre bundles, linear connections, and there are also seventy pages of notes, only a small part of which is merely references.

It is clear that Torretti himself is equally at home when dealing with science, and with history and philosophy. The question then is who will constitute the audience for his book. Certainly any research worker in relativity will have his understanding of what he is doing (as distinguished from his mere technical know-how) greatly deepened by reading it. The author, however, naturally seeks to cast his net wider. $\mathrm{He}$ imagines a reader equipped with "a clear recollection" of $n$-variable calculus and of linear algebra and of classical mechanics and electrodynamics. (Little philosophical knowledge is called for because the author supplies it as he goes along.) Such people will find the book hard going, but correspondingly rewarding.

C.W. Kilmister is Professor of Mathematics at King's College, University of London, and a past President of the British Society for the Philosophy of Science.

volume as a whole, which is forwardlooking but not trendy. The first part ("Processes") covers rock deformation, plate movements of many kinds, seismicity and magmatism. In the second part ("Case Histories") the effects of these processes are illustrated by reference to specific mountain belts, pride of place being given (fittingly, given the book's many Swiss links) to the Alpine-Himalayan belt.

Most of the articles are short and wellfocused; little space is wasted on the details of what is already known and the emphasis falls all the more clearly on what is still debatable. The displaced terrains - those small slabs of continental crust which migrated for thousands of kilometres before being trapped in a collision zone are discussed more than once. The stillunsolved problems of balancing structures observed in the upper crust against those inferred for the lower crust and lithospheric mantle are highlighted by a number of true-scale sections. Two excellent articles deal with the ductility of rocks and the mechanisms by which deformation takes place. There is a welcome lack of stereotyped "cartoons" and the vital but overworked arguments concerning crustal genesis have been given a well-earned rest.

The secret of success seems to be that the right authors have been asked to discuss the right questions. Collectively, they have produced a volume to which the general geologist can go not only for clear, pithy summaries of current problems but also for ideas and inspiration. It is a worthy tribute to two masters of European geology.

Janet V. Watson is a Professor of Geology at Imperial College, University of London. 